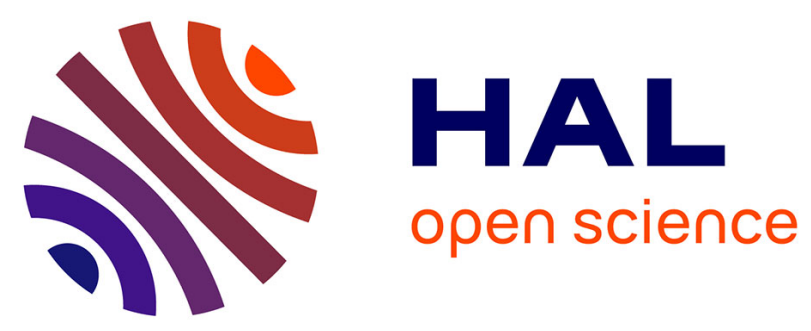

\title{
Expression des constantes de distorsion centrifuge des hexafluorures en fonction des fréquences harmoniques. Détermination de quelques constantes cubiques du potentiel
}

\author{
H. Berger, A. Aboumajd
}

\section{To cite this version:}

H. Berger, A. Aboumajd. Expression des constantes de distorsion centrifuge des hexafluorures en fonction des fréquences harmoniques. Détermination de quelques constantes cubiques du potentiel. Journal de Physique Lettres, 1981, 42 (3), pp.55-57. 10.1051/jphyslet:0198100420305500 . jpa00231872

\section{HAL Id: jpa-00231872 \\ https://hal.science/jpa-00231872}

Submitted on 1 Jan 1981

HAL is a multi-disciplinary open access archive for the deposit and dissemination of scientific research documents, whether they are published or not. The documents may come from teaching and research institutions in France or abroad, or from public or private research centers.
L'archive ouverte pluridisciplinaire HAL, est destinée au dépôt et à la diffusion de documents scientifiques de niveau recherche, publiés ou non, émanant des établissements d'enseignement et de recherche français ou étrangers, des laboratoires publics ou privés. 


\title{
LE JOURNAL DE PHYSIQUE-LETTRES
}

\section{Expression des constantes de distorsion centrifuge des hexafluorures en fonction des fréquences harmoniques. Détermination de quelques constantes cubiques du potentiel}

\author{
H. Berger et A. Aboumajd \\ Laboratoire de Spectronomie Moléculaire (*), Faculté des Sciences, 6, bd Gabriel, 21100 Dijon, France
}

(Reçu le 8 octobre 1980, accepté le 5 décembre 1980)

\begin{abstract}
Résumé. - Nous donnons les expressions des constantes de distorsion centrifuge des molécules $\mathrm{XY}_{6}$ en fonction des fréquences harmoniques; l'application est faite aux molécules $\mathrm{SF}_{6}$ et $\mathrm{UF}_{6}$. 4 constantes cubiques de $\mathrm{SF}_{6}$ ont été déterminées à partir des constantes rotationnelles.
\end{abstract}

Abstract. - We give for spherical $\mathrm{XY}_{6}$ molecules the expressions for the centrifugal distortion constants as a function of harmonic frequencies; application is made to $\mathrm{SF}_{6}$ and $\mathrm{UF}_{6}$.

4 cubic constants of $\mathrm{SF}_{6}$ have been calculated from the rotational constants.

1. Introduction. - Les molécules toupies sphériques de type $\mathrm{XY}_{6}$ n'ont pas de spectre de rotation pure et de ce fait les paramètres moléculaires de l'état vibrationnel fondamental ne peuvent être déterminés que de façon indirecte.

Pour la molécule $\mathrm{SF}_{6}$, la constante rotationnelle $B_{0}$ et la constante scalaire de distorsion centrifuge $D_{0}$ ont été déterminées à partir du spectre Raman de la bande $v_{2}$ [1]; la constante tensorielle de distorsion centrifuge $D_{\mathrm{t}}$ n'a été déterminée que très récemment par J. Bordé et al. [2] à partir du spectre d'absorption saturée de la bande $v_{3}$. Pour les autres hexafluorures, nous ne disposons pas à l'heure actuelle, de spectres à haute résolution qui nous permettraient d'effectuer des calculs analogues.

Il semble donc utile de chercher à exprimer les constantes spectroscopiques de l'état fondamental, et en particulier les constantes de distorsion centrifuge, en fonction des fréquences harmoniques de manière à déterminer leur valeur approchée. D'autre part, connaissant les constantes spectroscopiques, il est possible d'atteindre les constantes du potentiel anharmonique; nous indiquons quelques-unes des

(*) Equipe de Recherche associée au C.N.R.S. relations liant les constantes rotationnelles aux constantes cubiques de type $C_{1 q q}$.

2. Relations entre les constantes rotationnelles et les constantes de force pour une molécule $\mathrm{XY}_{6}$. $-\mathrm{Le}$ développement de l'hamiltonien deux fois transformé a été donné par Amat, Goldsmith et Nielsen [3] pour une molécule polyatomique quelconque. Hecht [4] et Moret-Bailly [5] ont simplifié le calcul des énergies en utilisant des opérateurs tensoriels sphériques dans la construction de l'hamiltonien effectif.

Il est donc souhaitable dans une étude rovibrationnelle de relier les coefficients du développement d'Amat et al. [3] à ceux de l'hamiltonien effectif.

Pour les molécules $\mathrm{XY}_{4}$ on peut trouver de telles relations dans les travaux de Hecht [4] et de Poussigue [6], mais pour une molécule $\mathrm{XY}_{6}$ des expressions analogues ne sont pas parues dans la littérature, sauf [7] pour deux constantes vibrationnelles $T_{33}$ et $G_{33}$ (notation de Hecht), relatives à la bande $v_{3}$.

Nous nous intéressons tout d'abord aux constantes rotationnelles de distorsion centrifuge intervenant dans l'état fondamental. En termes de tenseurs sphériques, l'opérateur en $P^{4}$ de l'hamiltonien $h_{2}^{+}$, s'exprime de la manière suivante $[4,6]$ : 


$$
\begin{aligned}
h_{2}^{+} \text {(rotation) } & =\Sigma_{\alpha \beta \gamma \delta}{ }^{\alpha \beta \gamma \delta} Y P_{\alpha} P_{\beta} P_{\gamma} P_{\delta} \\
& =\gamma_{0} \frac{P^{4}}{\hbar^{4}}+\varepsilon_{0} \frac{2 \sqrt{7}}{\sqrt{3}} \frac{1}{\hbar^{4}}\left[10 \Sigma_{\alpha} P_{\alpha}^{4}-6 P^{4}+2 \hbar^{2} P^{2}\right]+\Delta \beta \frac{P^{2}}{\hbar^{2}} \\
& =\frac{3}{16} \gamma_{0} \mathcal{H}_{\mathrm{A}_{1}}^{(0)}+\frac{1}{2} \sqrt{\frac{35}{2}} \varepsilon_{0} \mathcal{H}_{\mathrm{A}_{1}}^{(4)}-\frac{\sqrt{3}}{4} \Delta \beta \mathcal{C}_{\mathrm{A}_{1}}^{(0)} .
\end{aligned}
$$

Dans ces expressions les opérateurs $\mathcal{H}_{\mathrm{A}_{1}}^{(0)}, \mathfrak{H}_{\mathrm{A}_{1}}^{(4)}, \mathcal{C}_{\mathrm{A}_{1}}^{(0)}$ sont les tenseurs sphériques définis par MoretBailly [5]. Les constantes de distorsion centrifuge $\gamma_{0}$ et $\varepsilon_{0}$ (notation de Moret-Bailly) sont reliées aux constantes $D_{\mathrm{s}}$ et $D_{\mathrm{t}}$ introduites par Hecht [4] par les relations :

$$
\frac{\gamma_{0}}{h c}=-D_{\mathrm{s}}
$$

et

$$
\frac{\varepsilon_{0}}{h c}=-\frac{1}{2} \sqrt{\frac{3}{7}} D_{\mathrm{t}}=\frac{1}{2} \sqrt{\frac{3}{7}} t_{044} .
$$

En calculant les coefficients ${ }^{\alpha \beta \gamma \delta} Y$ et en identifiant les équations (1) et (3), on obtient alors les expressions suivantes :

$$
\begin{aligned}
\frac{\gamma_{0}}{h c} & =-\frac{8}{3} B_{\mathrm{e}}^{3}\left[\frac{1}{\omega_{1}^{2}}+\frac{1}{5 \omega_{2}^{2}}+\frac{3}{10 \omega_{5}^{2}}\right] \\
\frac{\varepsilon_{0}}{h c} & =\frac{\sqrt{3}}{10 \sqrt{7}} B_{\mathrm{e}}^{3}\left[\frac{1}{\omega_{5}^{2}}-\frac{1}{\omega_{2}^{2}}\right] \\
\frac{\Delta \beta}{h c} & =\frac{B_{\mathrm{e}}^{3}}{5}\left[\frac{2}{\omega_{2}^{2}}+\frac{3}{\omega_{5}^{2}}\right] .
\end{aligned}
$$

On remarquera que dans ces expressions, seules interviennent pour des raisons de symétrie les fréquences harmoniques $\omega_{1}, \omega_{2}, \omega_{5}$; elles correspondent aux bandes $v_{1}\left(\mathrm{~A}_{1 \mathrm{~g}}\right), v_{2}\left(\mathrm{E}_{\mathrm{g}}\right)$ et $v_{5}\left(\mathrm{~F}_{2 \mathrm{~g}}\right)$ permises en diffusion Raman.

La constante $\Delta \beta$ est une correction à la constante rotationnelle de l'état fondamental; en fait d'autres termes de l'hamiltonien $h_{2}^{+}$donnent une contribution plus importante :

$$
\begin{aligned}
B_{0}=\frac{\beta_{0}}{h c}=B_{\mathrm{e}}+\Delta & +\frac{1}{2} Y_{1}+Y_{2}+ \\
+ & \frac{3}{2}\left(Y_{3}+Y_{4}+Y_{5}+Y_{6}\right) .
\end{aligned}
$$

Les $Y_{i}$ (notation de Hecht) sont les constantes d'interaction vibration-rotation du second ordre. Elles ont une signification particulière, puisqu'elles correspondent à la différence entre les constantes rotationnelles de l'état excité et celles de l'état de base. Elles sont liées aux constantes cubiques du type $C_{1 q q}$ qu'il est possible de déterminer à partir des relations suivantes :

$$
\begin{aligned}
& Y_{1}=\frac{\beta_{1}-\beta_{0}}{h c}=4 \sqrt{3}\left(\frac{B_{\mathrm{e}}}{\omega_{1}}\right)^{3 / 2} C_{111}+\frac{4 B_{\mathrm{e}}^{2}}{\omega_{1}} \\
& Y_{2}=\frac{\beta_{2}-\beta_{0}}{h c}=\frac{4}{\sqrt{3}}\left(\frac{B_{\mathrm{e}}}{\omega_{1}}\right)^{3 / 2} C_{122}+B_{\mathrm{e}}^{2}\left(\frac{4 \omega_{2}}{\omega_{2}^{2}-\omega_{5}^{2}}\right) \\
& Y_{3}=\frac{\beta_{3}-\beta_{0}}{h c}=\frac{4}{\sqrt{3}}\left(\frac{B_{\mathrm{e}}}{\omega_{1}}\right)^{3 / 2} C_{133}+\frac{4 B_{\mathrm{e}}^{2}}{3 \omega_{3}}\left[\frac{\left(3 \omega_{3}^{2}+\omega_{4}^{2}\right)}{\omega_{3}^{2}-\omega_{4}^{2}} \zeta_{34}^{2}+\frac{\left(3 \omega_{3}^{2}+\omega_{6}^{2}\right)}{\omega_{3}^{2}-\omega_{6}^{2}} \zeta_{36}^{2}\right] \\
& Y_{4}=Y_{3}(3 \leftrightarrow 4) \\
& Y_{5}=\frac{\beta_{5}-\beta_{0}}{h c}=\frac{4}{\sqrt{3}}\left(\frac{B_{\mathrm{e}}}{\omega_{1}}\right)^{3 / 2} C_{155}+\frac{B_{\mathrm{e}}^{2}}{3 \omega_{5}}\left(\frac{\omega_{2}^{2}-9 \omega_{5}^{2}}{\omega_{2}^{2}-\omega_{5}^{2}}\right) \\
& Y_{6}=\frac{\beta_{6}-\beta_{0}}{h c}=\frac{4}{\sqrt{3}}\left(\frac{B_{\mathrm{e}}}{\omega_{1}}\right)^{3 / 2} C_{166}+\frac{4}{3} \frac{B_{\mathrm{e}}^{2}}{\omega_{6}}\left[\frac{\left(3 \omega_{6}^{2}+\omega_{3}^{2}\right)}{\omega_{6}^{2}-\omega_{3}^{2}} \zeta_{36}^{2}+\frac{\left(3 \omega_{6}^{2}+\omega_{4}^{2}\right)}{\omega_{6}^{2}-\omega_{4}^{2}} \zeta_{46}^{2}\right] .
\end{aligned}
$$

3. Applications à $\mathbf{S F}_{6}$ et $\left.\mathbf{U F}_{6} \cdot-a\right) \mathrm{SF}_{6}$. - Dans notre application à $\mathrm{SF}_{6}$ nous avons utilisé le champ de forces harmonique déterminé antérieurement par Aboumajd et al. [1], où les fréquences harmoniques ont les valeurs suivantes :

$$
\begin{array}{ll}
\omega_{1}=787 \pm 1 \mathrm{~cm}^{-1} ; & \omega_{2}=654 \pm 2 \mathrm{~cm}^{-1} ; \\
\omega_{5}=529 \pm 7 \mathrm{~cm}^{-1} ; & \left(B_{\mathrm{e}}=B_{0}\right) .
\end{array}
$$

Dans le tableau I, nous reportons les valeurs cal- 
Tableau I. - Constantes de distorsion centrifuge de $\mathrm{SF}_{6}$.

[SF $\mathrm{SF}_{6}$ centrifugal distortion constants.]

\begin{tabular}{ccc} 
& Valeur calculée & \multicolumn{2}{c}{$\begin{array}{c}\text { Valeur déduite } \\
\text { de l'expérience }\end{array}$} \\
& $-\bar{c}$ & $-\overline{\mathrm{c}}$ \\
$D_{\mathrm{s}}$ & $(6,36 \pm 0,07) \cdot 10^{-9} \mathrm{~cm}^{-1}$ & $(16 \pm 8) \cdot 10^{-9} \mathrm{~cm}^{-1}$ \\
$D_{\mathrm{t}}$ & $(-1,86 \pm 0,16) \cdot 10^{-10} \mathrm{~cm}^{-1}$ & $(-1,90 \pm 0,23) \cdot 10^{-10} \mathrm{~cm}^{-1}$
\end{tabular}

culées de $D_{\mathrm{s}}$ et $D_{\mathrm{t}}$ ainsi que celles déduites des analyses rovibrationnelles.

L'accord est excellent entre notre valeur calculée de $D_{\mathrm{t}}$ et celle déterminée par J. Bordé et al. [2], à partir du spectre d'absorption saturée de la bande $v_{3}$. Il n'en n'est pas de même pour la constante scalaire $D_{\mathrm{s}}$; nous pensons que cela est dû à la faible résolution du spectre Raman utilisé pour le calcul de $D_{\mathrm{s}}$. Il serait donc fort intéressant que la bande $v_{2}$ soit à nouveau étudiée en spectroscopie Raman cohérente (CARS ou Raman stimulé) de manière à augmenter de façon significative la résolution de la structure rotationnelle.

On peut, d'ores et déjà, supposer que notre valeur calculée, de $D_{\mathrm{s}}$, constitue une meilleure estimation de cette constante, que celle déduite de l'analyse Raman.

Bien qu'à l'heure actuelle, nous ne disposons pas de données expérimentales nombreuses sur la molécule $\mathrm{SF}_{6}$, il nous a été possible d'atteindre 4 des 22 constantes cubiques du potentiel à partir des relations (10-13). Les constantes $C_{111}$ et $C_{122}$ ont été calculées à partir des données Raman. La bande $v_{1}$, enregistrée en spectroscopie Raman conventionnelle [1] n'est pas résolue, mais par simulation du profil de cette bande nous avons trouvé une valeur approchée de la différence $B_{1}-B_{0}$. La constante $C_{122}$ est déduite de l'analyse rotationnelle de la bande $v_{2}$.

Les constantes $C_{133}$ et $C_{144}$ ont été obtenues à partir des spectres IR de $v_{3}$ [8] et $v_{4}$ [9]. Les coefficients de Coriolis $\zeta_{34}, \zeta_{36}$, et $\zeta_{46}$ apparaissant dans les équations (12) et (13), ont pu être calculés à partir des relations établies par Moret-Bailly [10], les reliant au coefficient $\zeta_{3}=0,6935$ issu de [8].

Tableau II. - Constantes cubiques de $\mathrm{SF}_{6}$ déterminées à partir des constantes rotationnelles.

$\left[\mathrm{SF}_{6}\right.$ cubic constants derived from rotational constants.]

$$
\begin{gathered}
\text { Différence } \\
\text { des constantes } \\
\text { rotationnelles } \\
\left(\mathrm{cm}^{-1}\right)
\end{gathered}
$$

$B_{1}-B_{0}=-8 \times 10^{-5}$

$B_{2}-B_{0}=5 \times 10^{-5}$

$B_{3}-B_{0}=-1,3 \times 10^{-4}$

$B_{4}-B_{0}=-2 \times 10^{-5}$

$\begin{array}{cc}\text { Références } & \begin{array}{c}\text { Constante } \\ \text { cubique } \\ \left(\mathrm{cm}^{-1}\right)\end{array} \\ \text { présent } & - \\ \text { article } & C_{111}=-14 \\ {[1]} & C_{122}=-32 \\ {[8]} & C_{133}=-56 \\ {[9]} & C_{144}=-3\end{array}$

b) ${ }^{238} \mathrm{UF}_{6}$. - Pour calculer les constantes de distorsion centrifuge $D_{\mathrm{s}}$ et $D_{\mathrm{t}}$ à partir des relations (4-7) nous avons utilisé les fréquences harmoniques données par McDowell et al. [11] :

$$
\begin{aligned}
\left(\omega_{1}=672 \pm 6 \mathrm{~cm}^{-1} ; \quad \omega_{2}=540 \pm 6 \mathrm{~cm}^{-1} ;\right. \\
\left.\omega_{5}=200 \pm 6 \mathrm{~cm}^{-1} ; \quad B_{\mathrm{e}} \sim 0,0555 \mathrm{~cm}^{-1}\right)
\end{aligned}
$$

Nous obtenons ainsi pour la première fois une valeur approchée de ces constantes :

$$
\begin{aligned}
& D_{\mathrm{s}}=(2,8 \pm 0,1) \cdot 10^{-9} \mathrm{~cm}^{-1} ; \\
& D_{\mathrm{t}}=(-7,3 \pm 0,6) \cdot 10^{-10} \mathrm{~cm}^{-1} .
\end{aligned}
$$

Nous pouvons alors prévoir la structure de l'état fondamental, qui compte tenu de la valéur de la constante $D_{v}$, devrait présenter un éclatement des niveaux beaucoup plus important que pour la molécule $\mathrm{SF}_{6}$.

En conclusion, les relations que nous avons établies devraient s'avérer fort utiles pour l'étude de molécules lourdes de type $\mathrm{XY}_{6}$, sur lesquelles les données sont inexistantes dans l'état fondamental.

Remerciements. - Les auteurs remercient MM. Pierre, Loete, Moret-Bailly, et Hilico pour leurs commentaires et suggestions sur ce travail.

\section{Bibliographie}

[1] Aboumad, A., Berger, H. and Saint-Loup, R., J. Mol. Spectrosc. 78 (1979) 486, 492.

[2] Bordé, J., Bordé, Ch. J., Salomon, C., van Lerberghe, A., Ouhayoun, M. and Cantrell, C. D., Phys. Rev. Lett. 45 (1980) 14, 17.

[3] Amat, G., Goldsmith, M. and Nielsen, H. H., J. Chem. Phys. 27 (1957) 838, 844.

Amat, G. and Nielsen, H. H., J. Chem. Phys. 27 (1957) 845, 850.

[4] Hecht, K. T., J. Mol. Spectrosc. 5 (1960) 355, 389.

[5] Moret-Bailly, J., Thèse, Cah. Phys. 15 (1961) 237.

[6] Poussigue, G., Thèse, Paris, C.N.R.S. n ${ }^{\circ}$ AO 2822.
[7] Jensen, C. C., Person, W. B., Krohn, B. J. and Overend, J., Opt. Commun. 2 (1977) 275, 279.

[8] Bordé, Ch. J., Ouhayoun, M., van Lerberghe, A., Salomon, C., Avrillier, S., Cantrell, C. D. and Bordé, J., Proceedings of the Fourth International Conference on Laser Spectroscopy, Ed. Wahlter and Rothe (Springer Verlag, Berlin).

[9] Kim, K. C., Person, W. B., Seitz, D. and Krohn, J. B., J. Mol. Spectrosc. 76 (1979) 322, 340.

[10] Moret-Bailly, J., Cah. Phys. 13 (1959) 476, 494.

[11] McDowell, R. S., Asprey, L. B. and Paine, R. T., J. Chem. Phys. 61 (1974) 3571, 3580. 